\title{
original
}

\section{Calidad sanitaria y mass media. El impacto mediático de la localización de notas de prensa sobre acreditación sanitaria en Andalucía}

\section{Palabras clave}

calidad de la atención de salud acreditación ciudadanía difusión de información

\author{
María Reyes León Vergara \\ Manuel Herrera Usagre \\ Rosario María Delgado Ramos \\ (Sevilla ,España) \\ Agencia de Calidad Sanitaria de Andalucía
}

\begin{abstract}
Resumen
El empleo de estrategias enfocadas a lograr el mayor impacto y eficacia de la comunicación debe ser un valor fundamental en cualquier organización, especialmente aquellas que orbitan en torno a la atención en salud. El presente trabajo realiza una valoración sobre el cambio de estrategia llevado a cabo en el año 2010 por el equipo de comunicación de la Agencia de Calidad Sanitaria de Andalucía, fundación pública andaluza encargada de la acreditación de calidad de los diferentes elementos del sistema sanitario. Tras el análisis de las notas de prensa emitidas, las noticias publicadas sobre acreditación de calidad y el recuento de centros y profesionales sanitarios acreditados se puede afirmar que ha habido un incremento significativo de noticias publicadas sobre acreditación de calidad en salud en el conjunto de la comunidad autónoma de Andalucía. Existen dos posibles factores a tener en cuenta. Por un lado, el mayor número de profesionales, centros y unidades que han apostado por la acreditación de calidad como una máxima de su quehacer profesional, fruto tanto del impulso que la administración sanitaria está dando a los programas de acreditación, como de la propia iniciativa de los profesionales. Por otro lado, existen indicios razonables para afirmar que, la localización de los actos y de la estrategia de comunicación de los mismos, enfatizando el contacto con los medios de comunicación locales, ha hecho que se multiplique la publicación de las notas de prensa en estos medios.
\end{abstract}




\section{Quality of Healthcare and Mass Media. Medial impact of Local Press Releases about Health Accreditation in Andalusia}

\begin{tabular}{c}
\hline Keywords \\
\hline quality of health care \\
accreditation \\
citizenship \\
information dissemination
\end{tabular}

\section{Cómo citar el artículo}

León Vergara, M. R.; Herrera Usagre, M. y Delgado Ramos, R. M. (2012). Calidad sanitaria y mass media. El impacto mediático de la localización de notas de prensa sobre acreditación sanitaria en Andalucía. Revista de Comunicación y Salud, 2(1), pp. 5-17. DOI: http://doi.org/10.35669/revistadecomunicacionysalud.2012.2(1).5-17

\section{Introducción}

Hoy en día, la difusión de información sobre salud en medios de comunicación está siendo concebida como una tarea indispensable, tanto como canal de información con la ciudadanía como de difusión de conocimientos científicos. El empleo de estrategias adecuadas hacia el mayor impacto y eficacia de la comunicación debe ser un valor fundamental en cualquier organización, especialmente aquellas que orbitan en torno a la atención en salud. No obstante, estas estrategias deben ser revisadas, evaluadas y mejoradas continuamente, dado el incesante cambio y evolución que el mundo de la comunicación experimenta en el contexto de la sociedad actual. El presente trabajo realiza una valoración sobre el cambio de estrategia llevado a cabo en el año 2010 por el equipo de comunicación de la Agencia de Calidad Sanitaria de Andalucía, fundación 
pública andaluza encargada de la acreditación de la calidad de los diferentes elementos que configuran el sistema sanitario. Se utilizarán diferentes indicadores, tanto de resultados productivos de la organización, del área de comunicación, como un indicador de impacto mediático de las noticias referentes a la tarea fundamental de la Agencia, la acreditación sanitaria.

Antes de comenzar a abordar los resultados del análisis descriptivo longitudinal, vamos a exponer someramente la tarea de la Agencia de Calidad Sanitaria de Andalucía, sus objetivos, así como algunos de sus indicadores fundamentales de resultados. De este modo, se contextualizará al lector sobre la importancia de la labor comunicativa de la organización. En segundo lugar, se expondrá la necesidad de transmitir y divulgar información en el contexto de la calidad. En tercer lugar, se explicará la importancia de desarrollar planes de comunicación eficaces en cualquier organización y, de manera particular, cuáles fueron las razones que motivaron el cambio de estrategia de comunicación en la Agencia de Calidad Sanitaria de Andalucía.

\subsection{La Agencia de Calidad Sanitaria: acreditación de calidad}

La Agencia de Calidad Sanitaria de Andalucía nace en octubre de 2002 en el marco del Modelo Andaluz de Calidad impulsado por la Consejería de Salud de la Junta de Andalucía en su I Plan de Calidad, y responde al compromiso social de ofrecer unos servicios de calidad que satisfagan las necesidades y expectativas de los ciudadanos.

Sus objetivos fundamentales, en función de los cuales dirige sus actuaciones, son el desarrollo del modelo de calidad de la Consejería de Salud en todos los centros y organizaciones sanitarias de Andalucía, el fomento de la cultura de la calidad y la seguridad en el sistema sanitario, el impulso de la mejora continua y la identificación de espacios de mejora, y la promoción de actuaciones que permitan la generación de conocimiento sobre calidad y su difusión e intercambio, para, como consecuencia de todo ello, incrementar la confianza de los ciudadanos hacia el Sistema Sanitario Andaluz.

Para conseguir estos objetivos, la Agencia de Calidad Sanitaria de Andalucía ha puesto en marcha diferentes programas de acreditación, que pretenden abarcar la realidad de los centros y unidades sanitarias, las competencias de los profesionales de la salud, la formación continuada y las páginas web de contenido sanitario.

Estos programas de acreditación de calidad arrojan en la actualidad cifras como los más de 500 centros o unidades sanitarias acreditados, fundamentalmente del sector público; los 3.718 profesionales que de manera individual han acreditado sus competencias; las más de 13.000 actividades de formación continuada y 761 programas de actividades certificados, y las 14 páginas web de contenido sanitario que han concluido favorablemente su proceso de acreditación (Agencia de Calidad Sanitaria de Andalucía, 2011).

Las cifras denotan la implicación de los profesionales de la sanidad pública andaluza en la acreditación sanitaria como una herramienta de mejora de la calidad de la asistencia. Por ello, es necesario comunicar a la sociedad que los centros, las unidades, los profesionales, se acreditan en calidad y que eso les beneficia positivamente en la atención que reciben. 


\subsection{Comunicar la calidad}

Precisamente la consideración del ciudadano como centro del sistema sanitario, inspirada por el paradigma de la atención centrada en el paciente (patient-centred care) (Byrne y Long, 1976; Laine y Davidoff, 1996; Mead y Bower, 2000), está siendo objeto de los planes de calidad de la administración sanitaria andaluza. En efecto, esta perspectiva estimula la tarea de comunicar la acreditación sanitaria como un logro con repercusión en los usuarios y pacientes. De hecho, un buen número de investigaciones se han dedicado a la medición del impacto de la acreditación sanitaria tanto en el área de centros hospitalarios (Salmon et al., 2003; Miller et al., 2005; Alkhenizan y Shaw, 2011; entre otros) como de profesionales (Peabody et al., 2008).

Pero también es importante poner en valor el esfuerzo de los profesionales de la salud que se han sometido a un proceso de evaluación voluntario en el que han tenido que medir su calidad a través de estándares o patrones de referencia a los que han debido responder mediante evidencias y pruebas del trabajo que realizan.

Así pues, la comunicación es indispensable en la difusión de estos logros de los profesionales que tienen una repercusión directa en los usuarios y pacientes de los servicios de salud acreditados y en la confianza que éstos tienen no sólo hacia sus centros de salud u hospitales más cercanos, sino hacia el sistema de salud en general.

Como indica Elena Becerra en su tesis doctoral Gestión de la comunicación en las instituciones sanitarias andaluzas (2006), "el interés por la calidad del servicio y la búsqueda de información en el paciente/usuario acercan a la institución, poco a poco, hacia la puesta en marcha de distintos canales de comunicación".

Es necesario comunicar la calidad de los servicios sanitarios como un elemento de trascendental importancia para que los servicios de salud generen confianza en los ciudadanos, de ahí el valor estratégico de las acciones de comunicación. Por otro lado, la ciudadanía demanda cada vez más noticias sanitarias y es responsabilidad de los medios ofrecer esa información "lo más rigurosamente posible consiguiendo así que sea veraz y de calidad para evitar la desconfianza, confusión, incertidumbre y alarma social” (Hernández-García, 2011: 29)

"Ninguna entidad importante existe hoy sin preocuparse de la comunicación, porque ésta se ha revelado como indispensable en un universo competitivo. El éxito de una institución, de un político, de un producto, está relacionado en proporción directa a la eficacia de su actividad comunicativa. [...] Y no cabe otra alternativa que comunicar" (Álvarez y Caballero, 1997: 19-20).

Hasta hace relativamente poco, la comunicación en el ámbito de la salud estaba relegada a la gestión de las crisis sanitarias y a amortiguar las informaciones negativas que aparecían en los medios de comunicación. En estos momentos, sin embargo, la comunicación en este campo tiene una actitud proactiva, abarcando una dimensión nueva como son las acciones de promoción y prevención de la salud y la difusión de los logros científicos, técnicos y profesionales que pueden mejorar la salud de la ciudadanía en general o de diferentes colectivos. 
Como recoge Virginia Silva (2001) en el artículo "Comunicación y salud" publicado en la revista Inmediaciones de la comunicación, "la comunicación para la salud abre las puertas y ventanas de los hospitales y consultorios y lleva los mensajes que pueden salvar vidas y aumentar el bienestar a los hogares y comunidades de todo el país".

Por tanto, la comunicación es en la actualidad una potente herramienta para cambiar comportamientos en la ciudadanía y ofrecer una visión positiva de los sistemas de salud. Para ello no sólo cambia el enfoque comunicativo de las informaciones sobre salud, sino también la propia concepción de la comunicación en sí, que posiciona al receptor, al destinatario del mensaje, como un ente activo (Holt y von Krogh, 2010).

\subsection{Plan de comunicación}

Informar sobre los procesos de certificación de calidad en el ámbito de los sistemas de salud es una tarea complicada para las instituciones que los impulsan, más aún cuando identifican, entre los públicos objetivos de sus acciones de comunicación, a la ciudadanía en general.

Generar interés sobre la acreditación en calidad y transmitir el valor de la misma son una labor aún más difícil, pero que, desde las instituciones de certificación públicas, como la Agencia de Calidad Sanitaria de Andalucía, se debe realizar como una parte importante del trabajo cotidiano.

Para que estos objetivos se cumplan, es necesario tomar conciencia de la importancia de mostrar a sus públicos su actividad, de hacer accesible su lenguaje sin renunciar al rigor, y de presentarse como una organización transparente, abierta a la sociedad. Pero, además, la institución debe contemplar la comunicación como un pilar básico y ha de contar con una planificación de ésta que ayude a conseguir sus objetivos estratégicos.

La elaboración de un plan de comunicación es imprescindible en toda organización, pero se hace más necesaria en aquellas cuyo trabajo es difícil de explicar. La identificación de unos objetivos de comunicación en consonancia con los generales de la organización, la delimitación de sus públicos, la puesta en marcha de acciones de comunicación efectivas, y la correcta evaluación de dichas acciones para comprobar su efectividad, son algunas de las cuestiones que debe contemplar un plan de comunicación.

Si se quiere llegar a los ciudadanos como usuarios y beneficiarios en última instancia de los sistemas de salud, se deben plantear acciones de comunicación cuyo impacto llegue directamente a este estrato de público.

En el caso que nos ocupa, la acreditación sanitaria por parte de la Agencia de Calidad Sanitaria de Andalucía, la convocatoria de actos de entrega de acreditación de calidad en los que estén presentes los medios de comunicación, la distinción a los profesionales sanitarios que se han acreditado, etc. pueden ser ejemplos de acciones que tengan repercusión en su entorno social y mediático. 
De ahí que en el año 2010, frente a una estrategia de comunicación inicial que se dirigía a los medios de comunicación de ámbito estatal o autonómico, se pasara a una estrategia de `ocalización' o lo que es lo mismo, se dirigieran los esfuerzos a reforzar la repercusión local de cada acreditación profesional o de centros o unidades. De hecho, desde hace años se lleva observando con atención un fenómeno aparentemente contradictorio en el mundo de la comunicación. Por un lado, los medios de comunicación de masas y las nuevas tecnologías nos proveen la oportunidad de acceder a más información de más lugares del mundo y, por otro lado, existe un creciente o, al menos, constante interés en las noticias de índole local (Silbermann, 1982).

De este modo, con la nueva estrategia, además de informar sobre las cifras globales de acreditación de competencias profesionales, se informa sobre el número concreto y las especialidades de los profesionales con sello de calidad de un centro específico, utilizando a los medios de comunicación locales como vehículo más eficaz para transmitir esa información a la ciudadanía. Este cambio de estrategia de comunicación vino dado por la escasa repercusión de los actos de entrega de distintivos de acreditación en los medios de comunicación de ámbito estatal y autonómico y por la voluntad de hacer llegar a la ciudadanía una información mucho más cercana sobre la calidad de los servicios sanitarios que recibe. En efecto, otras investigaciones han confirmado el efecto positivo que tienen los fenómenos de localización o hiperlocalización de medios de comunicación sobre la efectiva difusión de información en las comunidades locales (Baines, 2010).

Así, con posterioridad a estos actos, la redacción de una nota de prensa, la realización de fotos de dichos actos y el envío de este material a los medios de comunicación locales, además de los medios de ámbito estatal y autonómico, ayudan a la difusión de la acreditación de la calidad de los diferentes elementos del sistema sanitario en la ciudadanía. El número creciente de profesionales, centros y servicios que completan su acreditación en Andalucía implica una cifra importante de actos de entrega de certificados y distintivos. Unos actos que se convierten en acontecimientos de gran interés para los profesionales desde el punto de vista particular, pero que también sirven a la administración sanitaria para mostrar a la sociedad su apuesta por la calidad y por la mejora continua, convirtiéndose así en un potente instrumento de comunicación y transparencia del sistema sanitario con la ciudadanía.

El valor estratégico de estos actos se demuestra con la presencia en todos ellos de altos cargos de la administración autonómica sanitaria y de un buen número de profesionales del centro o unidad que ha completado su proceso de certificación de calidad, además de asociaciones de pacientes y medios de comunicación locales.

\section{Metodología}

El objetivo de este estudio se centra en describir la evolución de la aparición de noticias sobre acreditación de calidad entre 2008 y 2010 y explorar los posibles factores que han podido afectar a dicha evolución. Para ello, se ha acudido a tres fuentes de datos.

En primer lugar, el conjunto de notas de prensa emitidas por la Agencia de Calidad Sanitaria de Andalucía (ACSA a partir de ahora) durante el periodo 2008-2010 y que, en su inmensa 
mayoría, hacen mención de los procesos de acreditación sanitaria y sus actos de entrega.

En segundo lugar, se ha hecho una exploración sistemática de todas las noticias emitidas en medios de comunicación, entendiendo por éstos a la prensa escrita -tanto de carácter generalista como especializado-, la radio, la televisión y los medios digitales (páginas web y blogs). La estrategia para la búsqueda de estas noticias publicadas se desarrolla a partir de tres vías fundamentales:

1. Búsqueda en dossieres institucionales. La primera de ellas hace referencia a los dossieres de prensa diarios que se envían a ACSA desde la Consejería de Salud y que recogen la información sobre salud aparecida en la comunidad autónoma andaluza en medios de carácter generalista y algunos otros medios especializados. La periodicidad de estos dossieres es diaria y da cobertura de un amplísimo abanico de medios de comunicación, tanto del ámbito andaluz como nacional, haciéndose eco de las noticias relacionadas con la salud, la atención y la gestión sanitaria que suceden en Andalucía.

2. Búsqueda ampliada de feedbacks. Ésta tiene como instrumento fundamental la búsqueda a través de Google de la publicación de una determinada nota de prensa emitida. En otras palabras, buscamos aquellas comunicaciones de la Agencia de Calidad Sanitaria de Andalucía que rebotan en algún medio digital, ya sea prensa, televisión, radio o blogs especializados. En su mayoría, los medios generalistas -que no son medios especializados en materia sanitaria, sino que se hacen eco de la noticia por su interés general para sus lectores o audiencia- están localizados en el contexto de la comunidad autónoma andaluza y estimamos que su número esté en torno a los 67 . Además de los ubicados en nuestra comunidad autónoma, también aparecen en medios de otras comunidades, pero son una fracción muy pequeña. En cuanto a blogs especializados en materia sanitaria, éstos no tienen por qué estar ubicados en Andalucía y se trabaja con alrededor de una decena.

3. Búsqueda localizada. Como tercera vía, se procede a una búsqueda más localizada en algunos medios de comunicación en los que se tiene constancia o sospecha de la publicación de una determinada nota, bien por el interés que el propio medio ha manifestado, bien porque en el contexto de un acto de entrega de acreditación de calidad han estado presentes profesionales de algunos medios.

Como se hace notar en la estrategia, la mayoría de estas noticias publicadas parten de la redacción de notas de prensa de la propia Agencia de Calidad, haciendo de ésta una importante herramienta de difusión de su actividad, y que conforma un aspecto recogido en el plan de comunicación de la organización. Además, la cobertura de noticias se hace lo más amplia posible en cuanto a su cobertura territorial, pero lo más específica posible en su ámbito temático.

Finalmente, y como tercera y última fuente de datos, hemos utilizado el número total de acreditaciones de calidad, tanto de centros y unidades como de profesionales, que aporta la Agencia de Calidad Sanitaria de Andalucía en su página web (2011).

Para el análisis descriptivo que aquí presentamos hemos realizado una comparación entre, 
por un lado, el total de profesionales y centros acreditados por ACSA, así como las notas de prensa emitidas $y$, por otro, el número de apariciones en prensa. Para poder comparar ambos conjuntos de datos hemos utilizado el estadístico R2, como test de robustez, para la curva de regresión lineal que mide su aumento en el periodo de tiempo seleccionado. Con este dato, podremos hacer una aproximación a cómo se corresponden ambos datos. Por ejemplo, podremos observar cómo han aumentado el número de notas de prensa emitidas por la Agencia y cómo han aumentando el número de apariciones en prensa utilizando un estadístico de comparación. A continuación, procederemos al comentario de los resultados.

\section{Resultados}

A lo largo del periodo 2008-2010, espacio de tiempo analizado, la Agencia de Calidad Sanitaria de Andalucía ha emitido 202 notas de prensa, referidas, en su mayoría, a actos de entrega de certificación de calidad. De ellas, 55 se emitieron en 2008, 72 en 2009 y 75 en 2010 (Gráficos $1,2$ y 3$)$.

La búsqueda de las referencias publicadas a partir del envío de esas notas de prensa, realizada fundamentalmente vía internet a través de las ediciones digitales de los medios de comunicación y de Google, ha dado como resultado la aparición de 171 noticias sobre acreditación en 2008, 309 en 2009 y 506 en 2010. El total de profesionales acreditados pasó de 1.015 en 2008 a 1.393 en 2009 y a 3.587 en 2010. Por otro lado, el total de centros y unidades acreditadas pasó de 143 en 2008 a 208 en 2009 y a 506 en 2010.

Gráfico 1. Total de profesionales acreditados y número de apariciones en prensa

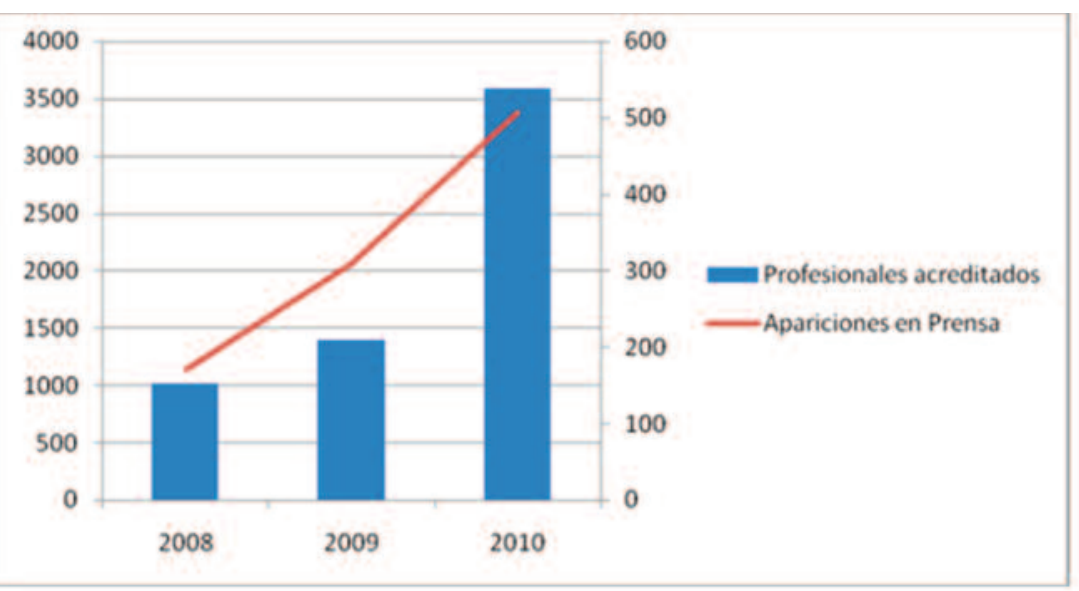

Fuente: Agencia de Calidad Sanitaria de Andalucía y elaboración propia. 
En el gráfico 1 se muestra la comparación entre el total de profesionales acreditados y el total de apariciones en prensa. Como se puede observar hay una correspondencia entre el crecimiento del número de profesionales acreditados (con el consecuente impacto de los actos de entrega de dicha acreditación) y las apariciones en prensa. De hecho, esta correlación se refuerza cuando acudimos a los estadísticos $R 2$ de ambos datos $(0,857$ para la variable profesionales acreditados y 0,989 para la variables apariciones en prensa).

Gráfico 2. Total de centros y unidades acreditadas y número de apariciones en prensa

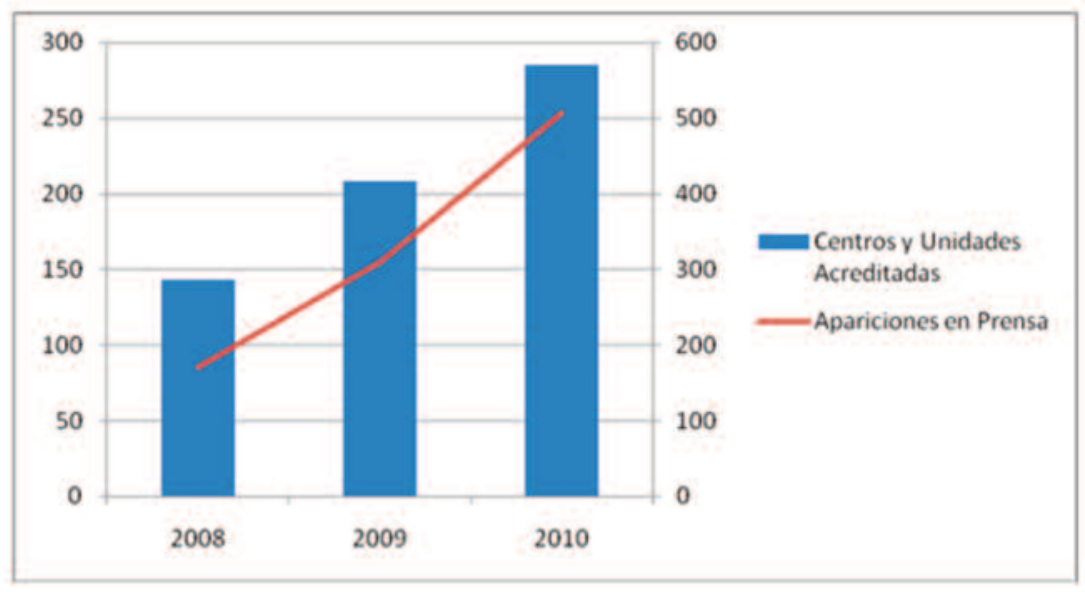

Fuente: Agencia de Calidad Sanitaria de Andalucía y elaboración propia.

En el gráfico 2 se muestra la comparación entre el total de centros y unidades acreditadas y el total de apariciones en prensa. Al igual que en el caso anterior, se observa una correspondencia entre el crecimiento del número de centros y unidades acreditadas (con el consecuente impacto de los actos de entrega de dicha acreditación) y las apariciones en prensa. En comparación con la anterior gráfica, esta correlación aparece más sólida cuando acudimos a los estadísticos R2 de ambos datos (0,997 para la variable centros y unidades acreditadas y 0,989 para la variables apariciones en prensa). Esta alta correlación a partir de las rectas de regresión lineal nos sugieren un mayor impacto mediático de los actos de acreditación de centros y unidades, siendo estos más numerosos que los de profesionales, celebrados bianualmente. 
Gráfico 3. Notas de prensa emitidas y número de apariciones en prensa

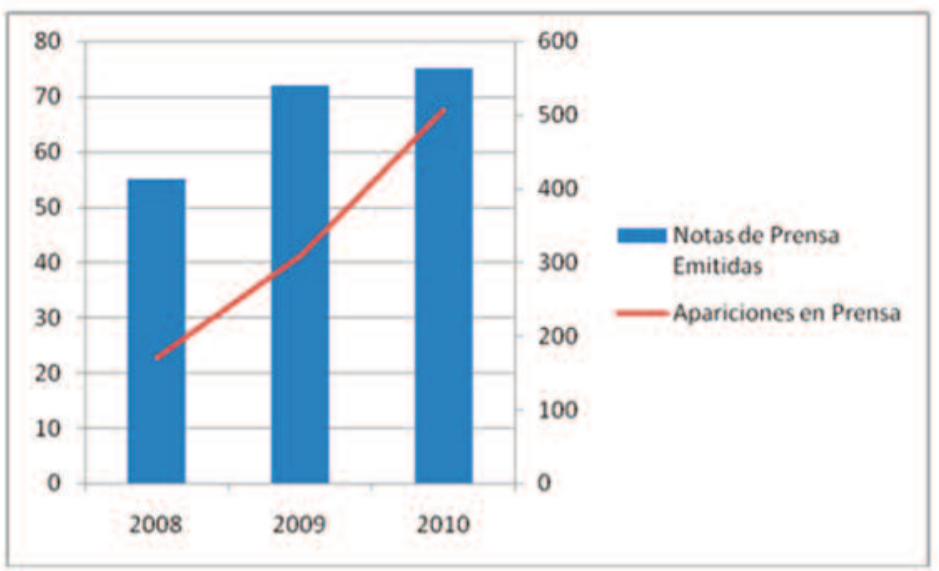

Fuente: Agencia de Calidad Sanitaria de Andalucía y elaboración propia.

Finalmente, en el gráfico 3 se muestra la comparación entre el total de notas de prensa emitidas por la Agencia y el total de apariciones en prensa. Como hemos venido observando, también surge una correspondencia entre el crecimiento del número de notas de prensa y las apariciones en prensa. No obstante, si bien el número de notas de prensa en el año 2009 creció sustancialmente respecto al año 2008, en el año 2010 la tendencia pareció estabilizarse. Si acudimos a la comparación entre los estadísticos R2 se observa también esa correlación, con resultados parecidos a los de la gráfica 1 (0,859 para la variable centros y unidades acreditadas y 0,989 para la variables apariciones en prensa). Para interpretar adecuadamente estos dos estadísticos, debemos tener en cuenta que el notable aumento de apariciones en prensa en el año 2010 y la estabilización de notas de prensa emitidas en el mismo año, controlan la inclinación de ambas rectas de regresión lineal y, por lo tanto, su test R2.

\section{Discusión y conclusiones}

Según los datos expuestos, se puede afirmar que ha habido un incremento significativo de noticias publicadas sobre acreditación de calidad en salud en el conjunto de la comunidad autónoma de Andalucía.

En el incremento de cifras existen dos posibles factores a tener en cuenta. Por un lado, el mayor número de profesionales, centros y unidades que han apostado por la acreditación de calidad como una máxima de su quehacer profesional, fruto tanto del impulso que la administración sanitaria está dando a los programas de acreditación, como a la propia iniciativa de los profesionales. Por otro lado, existen indicios razonables para afirmar que, la localización de los actos de entrega de distintivos de acreditación y la estrategia de comunicación de los mismos, enfatizando el contacto con los medios de comunicación locales, ha hecho que se multiplique la publicación de las notas de prensa en estos medios. 
El aumento de medios locales, bien a través de ediciones de medios de ámbito superior o de publicaciones exclusivamente municipales, reforzadas por la aparición de medios digitales de estas características, ha hecho que la acreditación de un centro de salud de un municipio sea una noticia de gran importancia para esa localidad en concreto. Es en este punto donde es importante reflexionar sobre si estas publicaciones en medios locales son un factor que desencadene el incremento de la confianza del ciudadano en su sistema sanitario.

Una cuestión que deja escaso margen a la duda hace referencia a que, los medios de comunicación locales, tanto prensa escrita como digital, como radio o televisión, son una herramienta para dar a conocer a la ciudadanía la calidad de los servicios de salud. Hacen que un concepto como la calidad se haga tangible y visible para la ciudadanía, configurando así una imagen positiva de los servicios de salud que reciben (Villafañe, 1993).De hecho, el interés por la acreditación que se manifiesta en la publicación de estas noticias, ofrecen indicios de la importancia de la obtención de un sello de calidad y su repercusión en la mejora de la atención sanitaria en su entorno.

En el presente artículo, una eventual limitación a la que nos enfrentamos como investigadores, ha sido la falta de desagregación de los datos de apariciones en prensa entre medios locales y medios supralocales. Este hecho, nos impide sacar conclusiones -sí avanzar indicios- sobre el impacto de lo que hemos denominado localización de las notas de prensa. Por otro lado, al ser una investigación descriptiva, tampoco podemos atribuir de forma categórica a la localización de las notas de prensa el que sea la causa del aumento de los impactos (podría haber otras causas, variables confusoras, contextos mediáticos diferentes, etc.) a falta de análisis multivariables cuando se tenga una masa crítica suficiente de datos. Como bien se dice, sí se puede afirmar que hay indicios de su impacto positivo. Por tanto, abogamos por la continuidad de otros estudios futuros que, tal y como hace Hernández García (2011), pudiesen avanzar en la relación entre las estrategias de comunicación y el aumento de impacto en los medios locales, explorando así los efectos que eso ha provocado en la mejora del conocimiento de marca de la Agencia, de su prestigio, etc. Nuestras presentes líneas de investigación van en esa dirección.

En definitiva, este trabajo ha presentado indicios sobre el impacto positivo de una estrategia de comunicación que combina, tanto la tarea fundamental de emisión de notas de prensa, como la focalización de noticias referentes a la acreditación de centros, unidades clínicas y/o profesionales hacia aquellas comunidades locales que son atendidas por éstos. En otras palabras, permitimos que la ciudadanía conozca que su centro sanitario más cercano o los profesionales de la salud que la atienden cuentan con una distinción que reconoce el esfuerzo en pro de la calidad y de la mejora continua. Faltaría conocer, y es ése el reto de eventuales estudios futuros, si ese conocimiento de la acreditación de calidad de un servicio de salud supone una mayor confianza en el mismo. 
Referencias

Agencia de Calidad Sanitaria de Andalucía. (2011). Resultados de Acreditación. Disponible en http://www2.acsa.junta-andalucia.es/agenciadecalidadsanitaria/resultados_de_acreditacion. Recuperado el 9 de noviembre de 2011.

Alkhenizan, Abdullah, y Shaw, Charles. (2011). Impact of Accreditation on the Quality of Healthcare Services: a Systematic Review of the Literature. Annals of Saudi Medicine. Jul-Aug; vol. 31, n 4, pp. 407-416.

Álvarez Domínguez, Tomás y Caballero Hueso, Mercedes. (1997). Vendedores de imagen: los retos de los nuevos gabinetes de comunicación. Barcelona: Paidós.

Baines, David. (2010). Hyper-local: Glocalised rural news. International Journal of Sociology and Social Policy. Vol. 30, nº 9-10, pp. 581-592. doi: 10.1108/01443331011072316

Becerra Muñoz, Elena. (2006). Gestión de la comunicación en las instituciones sanitarias andaluzas. Tesis doctoral. Málaga: Universidad de Málaga.

Byrne, Patrick Sarsfield y Long, Barrie E. L. (1976). Doctors Talking to Patients. London: HMSO.

González Borjas, Antonia. (2000). El fenómeno de la 'edicionalización' en la prensa local. Revista Latina de Comunicación Social Vol. 31, n 3-4. Disponible en:

http://www.ull.es/publicaciones/latina/aa2000kjl/z31jl/85borjas.htm. Recuperado el 5 de noviembre de 2011.

Hernández García, Laura. (2011). Análisis de los contenidos sociosanitarios en prensa local. Revista Española de Comunicación en Salud. Vol. 2, nº 1, pp. 24-31.

Holt, Kristoffer y von Krogh, Torbjörn. (2010). The Citizen as Media Critic in Periods of Media Change. Observatorio (OBS*). Vol. 4, $n^{\circ}$ 4, pp. 287-306. Disponible en http://obs.obercom.pt/index.php/obs/index. Recuperado el 9 de noviembre de 2011.

Laine, Cristine y Davidoff, Frank. (1996). Patient-Centered Medicine: a Professional Evolution. Journal of the American Medical Association. Vol. 275, pp. 152-156.

doi: 10.1001/jama.1996.03530260066035

Miller, Marlene R. et al. (2005). Relationship Between Performance Measurement and Accreditation: Implications for Quality of Care and Patient Safety. American Journal of Medical Quality. Vol. 20, pp. 239-252. doi: 10.1177/1062860605277076

Mead, Nicolas y Bower, Peter. (2000). Patient-Centredness: A Conceptual Framework and Review of the Empirical Literature. Social Science and Medicine. Vol. 51, no. 7, pp. 1087-1110. doi: 10.1016/S0277-9536(00)00098-8 
Peabody, John W. et al. (2008). Should we Have Confidence if a Physician is Accredited? A Study of the Relative Impacts of Accreditation and Insurance Payments on Quality of Care in the Philippines. Social Science \& Medicine. Vol. 67, n 4, pp. 505-510.

doi: 10.1016/j.socscimed.2008.04.013

Salmon, J. Warren; Heavens, John; Lombard, Carl y Tavrow, Paula. (2003). The Impact of Accreditation on the Quality of Hospital Care: KwaZulu-Natal Province, Republic of South Africa. Operations Research Results 2(17), Bethesda, MD. Publicado por US Agency for International Development (USAID) by the Quality Assurance Project, University Research Co., LLC.

Silva Pintos, Virginia. (2001). Comunicación y salud. Inmediaciones de la comunicación. №3, pp. 121-136.

Silbermann, Alphons. (1982). Overriding Problems of Local Communication. Communications. Vol. 8, n³, pp. 265-274.

Villafañe, Justo. (1993). Imagen Positiva. Gestión estratégica de la imagen en la empresa. Madrid: Pirámide. 